\title{
Toward an Accurate Scaling Relation for the Critical Current in Niobium-Tin Conductors
}

\author{
Arno Godeke, Bennie Ten Haken, and Herman H. J. Ten Kate
}

\begin{abstract}
Until a few years ago, a set of equations commonly referred to as the Summers relations gave the most accurate description of the critical current in $\mathrm{Nb}_{3}$ Sn conductors as a function of applied field, temperature and axial strain. Although highly empirical, they describe reasonably well the critical current data of past $\mathrm{Nb}_{3} \mathrm{Sn}$ conductors. New data from various types of $\mathrm{Nb}_{3} \mathrm{Sn}$ conductors, as well as recent analysis of the ITER CS model coil results reveal however, that this description lacks the precision, required to correlate the conductor data to the model coil results. This discrepancy, attributed to the highly empirical background for the relations, manifests itself mainly in the strain- and temperature dependence. The development of an alternative, more accurate description of the behavior of the critical current, starting from a more fundamental description of the strain dependence, has been initiated. At the moment, the development concentrates around the improvement of the temperature dependency relations to achieve a better accuracy of the overall descriptions, especially in the high temperature region. This contribution gives an overview of the latest results.
\end{abstract}

Index Terms-Critical current, deformation, field, Niobium-tin, relation, scaling, strain, temperature.

\section{INTRODUCTION}

A $\mathrm{N}$ IMPROVED overall scaling equation, which included a three-dimensional deformation description, as well as a small improvement in the temperature accuracy in the form of two deviating constants, has been proposed recently [1]-[4]. This publication concentrates on the accuracy analysis of this new description and the steps that have to be made to further improve the temperature dependent part. The equations themselves have been extensively published in the references above and will not be repeated here.

\section{ANALysis of Full Size ITER COIL Results}

The recent results on full size model coils raises the question to what extend these systems reach their design goals. The question whether such a magnet performs within expectations is only useful when an accurate correlation can be made between the measured coil performance and the specifications of the components with which it was constructed (e.g., cables, sub-cables, or in a deeper decomposition into strands).

The performance of the model-coil systems is currently verified via critical temperature $\left(T_{c}\right)$ values, derived from voltagetemperature transitions at a fixed current $(I)$, and from critical

Manuscript received September 24, 2001.

The authors are with the University of Twente, Department of Applied Physics, Low Temperature Division, Enschede, The Netherlands (e-mail a.godeke@ tn.utwente.nl).

Publisher Item Identifier S 1051-8223(02)03881-2. current $\left(I_{c}\right)$ or quench current $\left(I_{q}\right)$ measurements at a fixed temperature $(T)$. A third verification parameter is the estimate of the conductor deformation state, which is derived from numerical mechanical models.

From the conductor side, a huge conductor database is available, mainly from the worldwide conductor specification measurements with contributions from various laboratories and institutes. These data consist of $I_{c}$-values versus $T$, magnetic induction $(B)$ and axial conductor deformation.

Recently a first serious attempt was made to relate the experimental coil data to the conductor data base by applying a set of scaling relations, commonly referred to as the "Summers" relations [5]. For single sample measurement descriptions, these relations appear to be sufficiently accurate. Analysis of the model coil results have shown however, that for description of the large amount of conductor data with one single set of fixed parameters per conductor type, as well as the coupling to the model coil results, lack accuracy. Although part of the discrepancy arises from clear inconsistency in the conductor database itself, the descriptions also exhibit inherent inaccuracy, due to the one-dimensional character of the deformation description and to the imperfect temperature dependency relations, especially in the region close to $T_{c}$.

\section{Characterization OF StRANDS}

Approximately 50 samples, from 6 manufacturers were analyzed at the University of Twente in the frame of a world-wide strand characterization sequence. The samples were measured for $I_{c}$ as function of $B, T$ and axial strain $(\varepsilon)$ on two different holders, described in [3] and [6]. $I_{c}$-values were measured in the range $1 \mathrm{~T} \leq B_{\text {applied }} \leq 13 \mathrm{~T}, 4.23 \leq T \leq 18 \mathrm{~K}$ and $-0.7 \%$ $\leq \varepsilon_{\text {total }} \leq+0.4 \%$, which resulted in $I_{c}$-values ranging from 0 A to $>1000 \mathrm{~A}$. Typical results for $I_{c}(B, T)$ and $I_{c}(B, T, \varepsilon)$ are shown in Fig. 1. Samples produced by Furukawa are selected as an example in this publication, but other conductor types show similar behavior. The results of the measurements were carefully analyzed and related to the improved scaling relations. A clear separation between material- and conductor dependent scaling parameters can be made [1]-[3] and it is possible to describe the behavior of all samples from each manufacturer, measured in two different experimental setups, with one single set of parameters.

The only parameter that is allowed to vary, and only per conductor type and experimental setup, is the pinning related overall pre-factor $(C)$, which is also proportional to the superconducting cross-section. Variation of $C$ is only allowed when exactly identical samples in the same setup exhibit clearly a variation in measured $I_{c}$-values. The overall accuracy across 

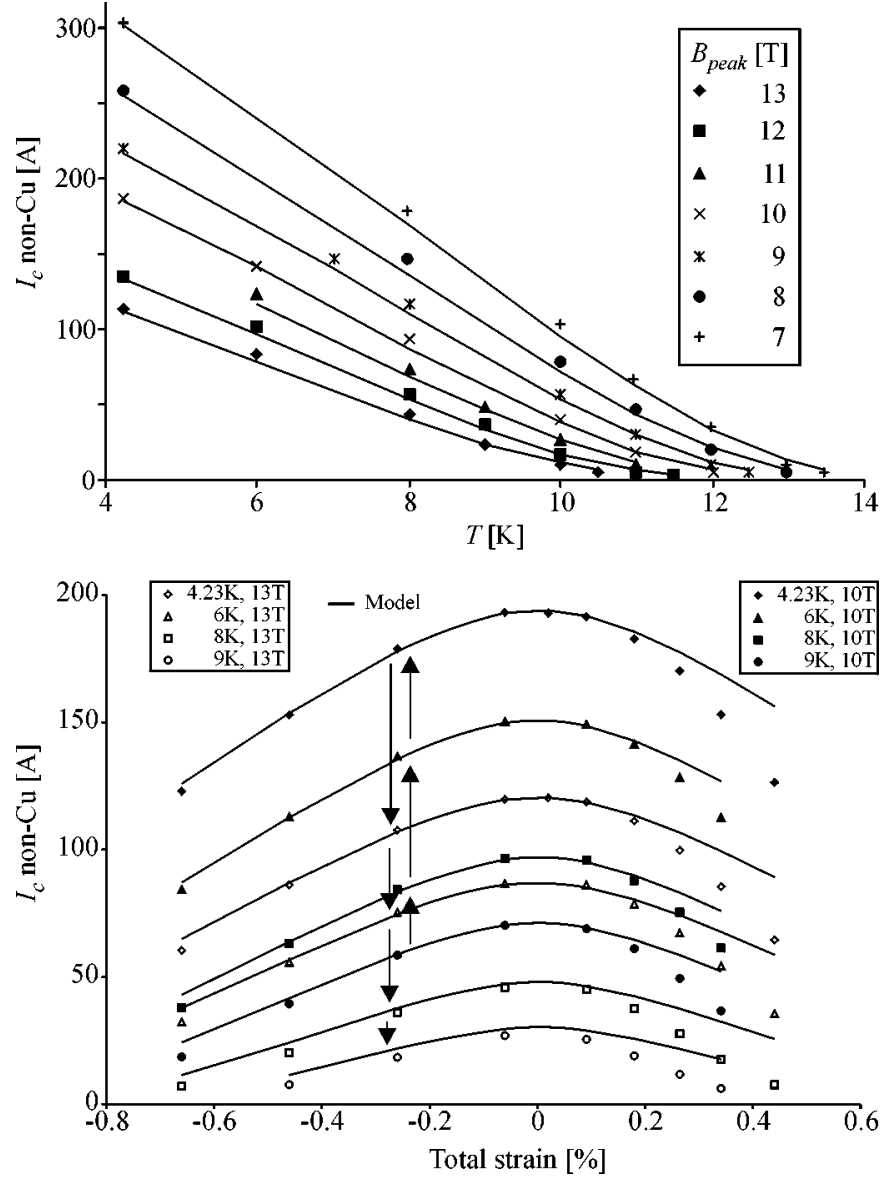

Fig. 1. Typical results of the $I_{c}(B, T, \varepsilon=0.27 \%)$ measurements (top) and the $I_{c}(B, T, \varepsilon)$ measurements for Furukawa samples. The points are measured and the lines are calculated with the improved scaling relations and one single set of parameters. The point at which the conductor starts to deform plastically cannot be described by the used elastic 3-D deformation model without taking into account changing Poisson ratios.

The arrows in the lower plot indicate the order of measurement, i.e., at each strain value, a field and temperature sweep is made, before the strain is adjusted to the following strain. $B_{\text {peak }}$ indicates the maximum of the applied field plus self-field at any place in the conductor, between the voltage connections.

the entire $B, T$ and deformation range has a standard deviation of $10 \mathrm{~A}$, and a maximum local error of 40-70 A (Table I).

\section{A. The Influence of a Parallel Resistive Current}

The current flowing parallel to the superconducting filaments, through the normal conducting matrix and/or sample holder, should be accurately taken into account. A certain voltage across the sample will result in some current flowing in the normal conducting materials, and its influence, which is often underestimated, should be carefully taken into account. At high $I_{c}$-criterions (e.g., 100 and $500 \mu \mathrm{V} / \mathrm{m}$ ), where this parallel resistive current can be of the order of amperes and thus certainly no longer negligible. These parallel resistive currents are determined by measurement of the resistance versus field, just above $T_{c}$. This result is subtracted from the uncorrected measured overall " $I_{c}$," resulting in the $I_{c-\text { nonCu }}$ of the superconductor $\left(I_{c \text {-nonCu }}=I_{\text {overall }}-I_{\text {resistive }}\right)$.
TABLE I

OVERVIEW OF THE SCALING ACCURACIES

\begin{tabular}{llcccc}
\hline & Criterion: & \multicolumn{2}{c}{$500 \mu \mathrm{V} / \mathrm{m}$} & $100 \mu \mathrm{V} / \mathrm{m}$ & $10 \mu \mathrm{V} / \mathrm{m}$ \\
\hline Range: & Setup: & Strain & Barrel & Barrel & Barrel \\
\hline \multirow{3}{*}{ C [AT] } & 9298 & 9451 & 9148 & 8632 \\
Overall & STDEV [A] & 9.4 & 11 & 11 & 9 \\
& Max. Local [A] & 41.7 & 70 & 64 & 51 \\
\hline \multirow{2}{*}{$\geq 5 \mathrm{~T}$} & C [AT] & 9298 & 9930 & 9566 & 8950 \\
& STDEV [A] & 3.4 & 3 & 2 & 2 \\
& Max. Local [A] & 15.7 & 6 & 5 & 4 \\
\hline
\end{tabular}

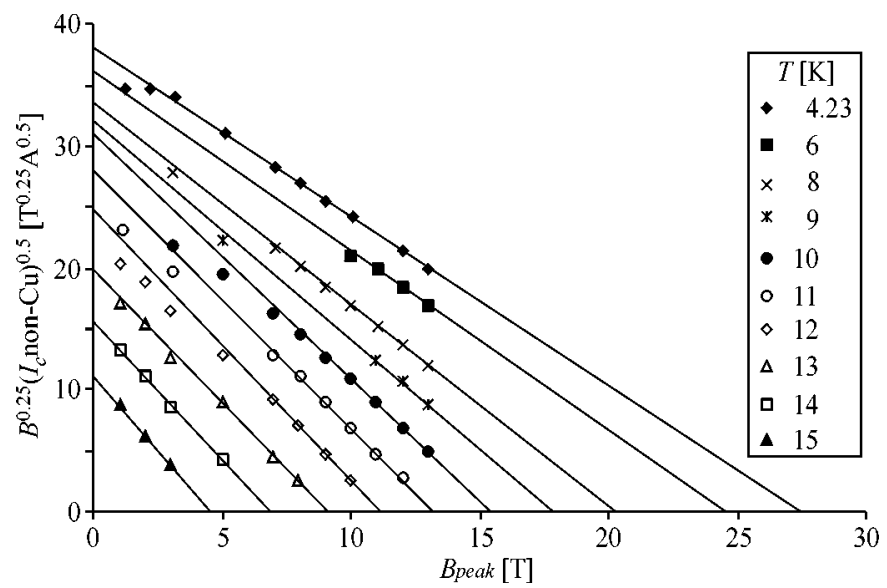

Fig. 2. Typical "Kramer" extrapolations to $B_{c 2}(T, \varepsilon=0.27 \%)$ for a Furukawa conductor.

\section{B. The Influence of Self-Field Corrections}

Apart from proper corrections for the parallel resistive current, also the self-field has to be taken into account. A dramatic improvement in accuracy occurs if only data equal to or above $5 \mathrm{~T}$ are considered in the overall scaling (see Table I). At first impression this can be attributed to nonvalidity of the "Kramer"-pinning relations in the low field range [7] and [8], but since deviations between model and measurement occur mainly at high currents (see Fig. 2), it is probably due to incorrect (i.e., too low) self-field calculations in the sample. This has been confirmed by comparison of low field magnetization measurements and Kramer extrapolations to low field from high field measurements [9]. This implies that the Kramer pinning relations are valid down to about $1 \mathrm{~T}$, which is in contrast to earlier publications [7] and [8]. In a recent publication [10], a new model is proposed for the description of $J_{c}(B, T)$, which also results in a Kramer field dependence, valid down to a low field value (depending on the choice of fitting parameters) of approximately $2 \mathrm{~T}$. Comparison of this description with magnetization measurements, also confirmed a validity range for the Kramer relation down to approximately $1.5 \mathrm{~T}$.

\section{The Influence of Various Voltage Criterions}

Another important conclusion from Table I is that it is possible to describe $I_{c}$ at different voltage criteria by changing only the overall pinning related pre-constant $C$ in the scaling relations, provided the $V(I)$-curves behave according to a power law with a constant $n$-value. All measured samples can be described with such a relation, provided that proper corrections are made for common measuring errors (see below). The fact that 


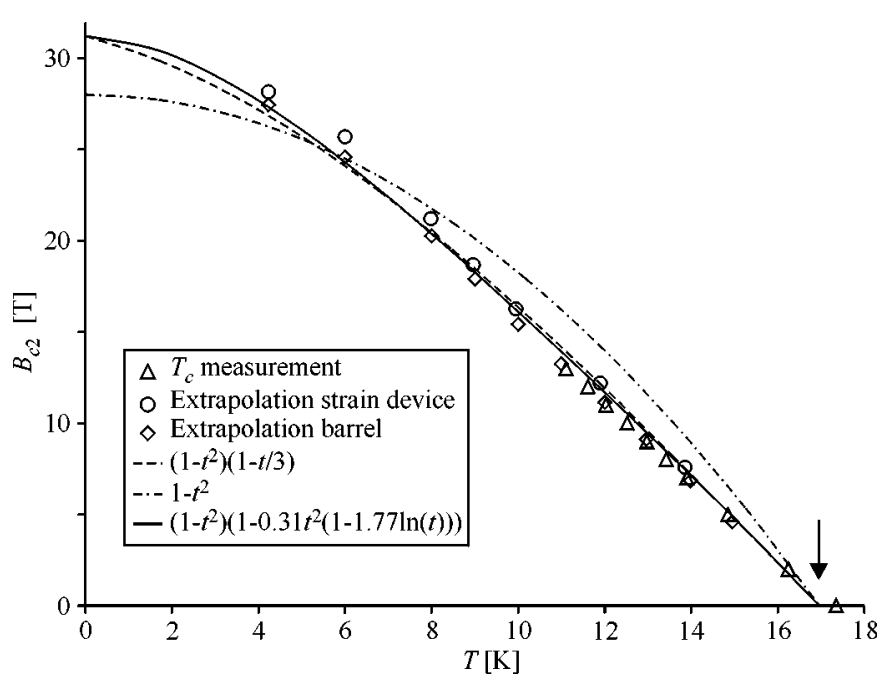

Fig. 3. Typical extrapolated and directly measured $B_{c 2}(T)$ data and possible descriptions for a Furukawa conductor. Points are measured and lines are calculated by the model. The arrow indicates a small "tail" in the $B_{c 2}(T)$ behavior possibly caused by $T_{c}$-inhomogeneities across the sample, which might be very difficult to take into account in an accurate description.

the $n$-value is a strong function of $B$ and $T$ has no significant effect.

\section{The Influence of Non-Zero Measuring Currents}

Another error source originates from the fact that the critical parameters in the scaling relations are defined at zero current through the superconductor. During measurements there is however always a current to create a measurable signal. Its influence will be discussed in the next section.

\section{The Temperature DePENDENCY}

The accuracy of the temperature dependent part of the descriptions is determined by the accuracy of the $B_{c 2}(T)$ critical line and the way this is implemented in the overall scaling relation. Typical $B_{c 2}(T)$ curves are shown in Fig. 3

The points in Fig. 3 are determined in three different ways; "Kramer"-extrapolation to $I_{c}=0$ from a deformation experiment, "Kramer"-extrapolation to $I_{c}=0$ from a long sample (ITER "barrel") setup (see Fig. 2) and directly measured by a resistive $T_{c}$-measurement on a barrel at a current of $0.5 \mathrm{~A}$ through the sample. The lines are calculated from the overall description after data-fits to all measured critical current values. Three different descriptions are presented for $B_{c 2}(t) / B_{c 2}(0)$ (in which $t$ represents the reduced temperature $T / T_{c}$ ):

- $1-t^{2}$ : Follows from thermodynamic considerations.

- $\left(1-t^{2}\right)\left(1-0.31 t^{2}(1-1.77 \ln (t))\right)$ : The adjusted thermodynamic form as proposed by Summers et al. [1] which causes effectively a more linear behavior at higher temperatures.

- $\left(1-t^{2}\right)(1-t / 3)$ : A simplified alternative for the Summers form, adopted by the ITER community.

It is clear that the thermodynamic form does not correspond to the data. The third form has the disadvantage that it shows a nonzero derivative at $T=0 \mathrm{~K}$, which is physically incorrect.

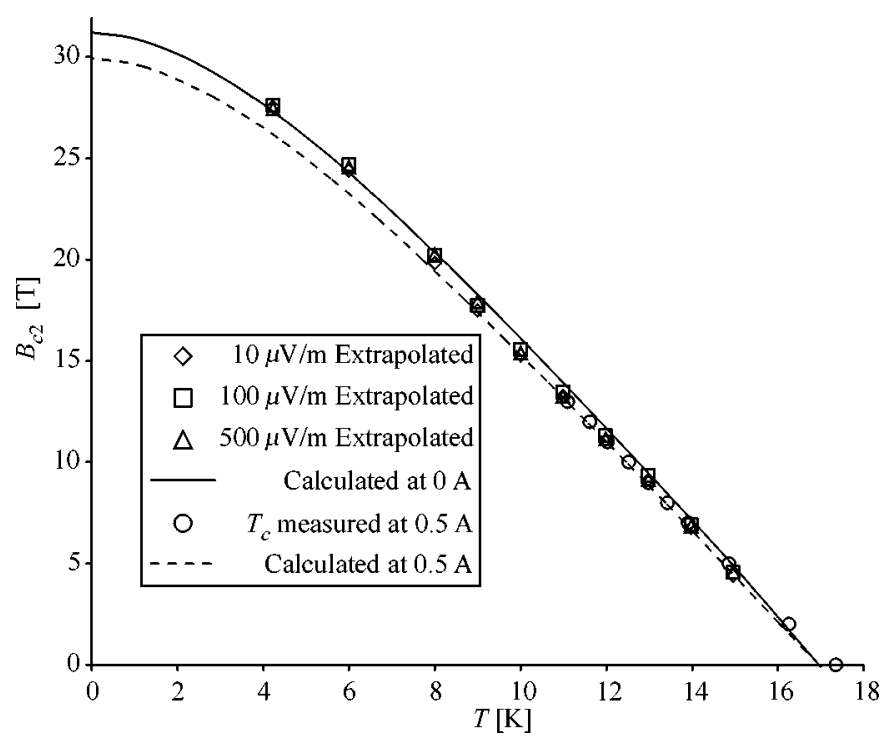

Fig. 4. Model (lines) and measured (points) $B_{c 2}(T)$ behavior for a Furukawa conductor on an ITER barrel. The extrapolated points from the strain device measurements are omitted for clarity.

Although fully empirical, the Summers form is reasonably accurate and the best alternative so far to be used in the overall scaling relations.

The deviations in the points from the strain device measurements are attributed to the following possible sources of errors. The strain device as well as the barrel are constructed from Ti-6Al-4V, but have a different shape and mounting procedure, which could lead to a slightly different strain and thus a small shift in the extrapolated $B_{c 2}(T)$ points.

The calculated lines follow from data-fits over all measured $I_{c^{-}}$-values. Much more data are extracted from the barrel in comparison to the strain device and hence the model lines will be closer to the barrel points. The largest and most consistent data set is available from the measurements on the barrel. These data, (the extrapolated $B_{c 2}$ values and measured $T_{c} \mathrm{~s}$ ) lie systematically below the calculated line. As mentioned before, the model line is defined at zero measuring current. If the line is adjusted to 0.5 A measuring current, as is presented in Fig. 4, it turns out that the shift that occurs for high temperatures exactly cancels the deviation which is present for the zero current calculation.

To verify the $B_{c 2}(T)$ description for the complete temperature range, a high field resistive $T_{c}$ measurement at a known measuring current would be preferable, since for the extrapolated values from $I_{c}$ measurements it is very hard, if not impossible, to get an indication of the influence of the nonzero measuring current. Moreover it can be seen that even changing the $I_{c}$-criterion (which effectively changes the measuring current) has negligible influence on the extrapolated $B_{c 2}(T)$ points. Considering the shape of the calculated lines with respect to the data points, it is clear that the best temperature dependency description (Summers) still lacks precision.

Table II is a calculated estimate of the large errors that can be made in the determination of the critical parameters to be used in the scaling relations, if they result from experiments with nonzero measuring currents. 
TABLE II

Calculated Influence of a Non-Zero Measuring CuRRENT ON THE CRitical Parameters of a Furukawa CONDUCTOR

\begin{tabular}{lccccc}
\hline & $I=1 \mathrm{~A}$ & $I=0.5 \mathrm{~A}$ & $I=0.05 \mathrm{~A}$ & $I=0.005 \mathrm{~A}$ & $I=0 \mathrm{~A}$ \\
\hline$T_{c}$ at $B=10 \mathrm{~T}[\mathrm{~K}]$ & 12.34 & 12.46 & 12.66 & 12.72 & 12.74 \\
$B_{c 2}$ at $T=10 \mathrm{~K}[\mathrm{~T}]$ & 14.97 & 15.30 & 15.85 & 16.03 & 16.11 \\
\hline
\end{tabular}

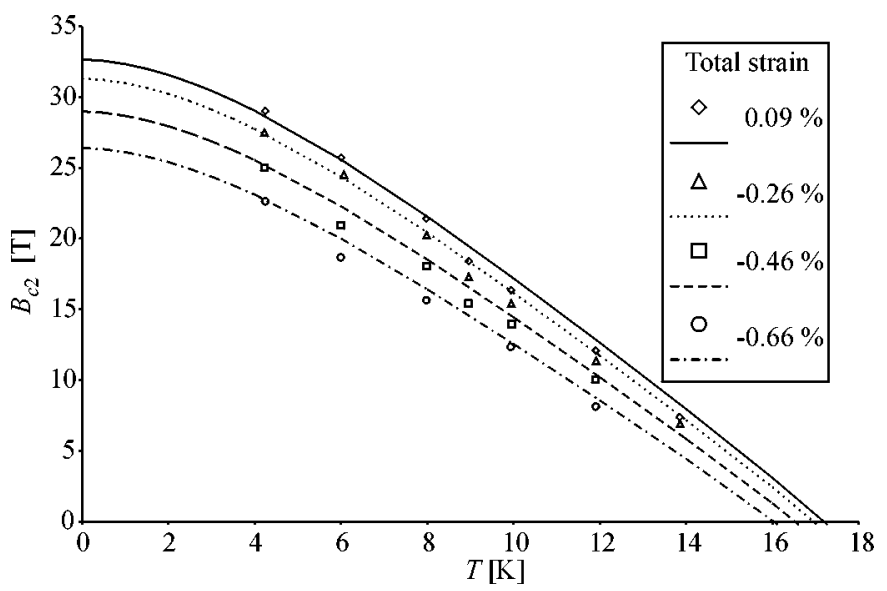

Fig. 5. Extrapolated (points) and calculated (lines) variation of $B_{c 2}(T)$ with deformation.

\section{VARIATION OF THE TEMPERATURE DEPENDENCY WITH STRAIN}

The ratio between the amount of variation of $B_{c 2}$ and $T_{c}$ with strain is represented in the scaling relations by a power of 3 [11], [12], i.e., the relative change in $B_{c 2}$ is a factor of 3 larger than the relative change in $T_{c}$.

From the measurements on the strain device, extrapolated $B_{c 2}(T)$ points at various strain values can easily be deduced. Fig. 5 shows some of these points, together with the model dependencies. There is a large amount of noise in the extrapolated points, due to the limited amount of data to extrapolate from, and due to the fact that the regulated temperatures are not always exactly the same, which is caused by the order of the measurements (see Fig. 1). But apart from the noise, Fig. 5 is a strong indication that over the complete range, the description is correct.

\section{CONCLUSIONS}

- If only fields $\geq 5 \mathrm{~T}$ are considered in the $I_{c}(B, T, \varepsilon)$ model, the accuracy is extremely high. The inaccuracies typically amount to a standard deviation of 3 A with a maximum local error of $10 \mathrm{~A}$. Lower field accuracy can probably be increased by improving the self-field corrections.

- It is possible to describe various $I_{c}$-criteria, by variation of only the overall pre-constant, provided that measurements are properly corrected, and the $V(I)$ transitions strictly follow a power law.

- The temperature dependency description exhibits a small deviation but has no fundamental background. Ideal would be a more fundamentally based description, leading to an improvement in accuracy.

- Very large errors in the determination of the critical parameters will occur when the influence of both the measuring current and parallel resistive current magnitudes, are not properly taken into account.

\section{REFERENCES}

[1] A. Godeke, B. ten Haken, and H. H. J. ten Kate, "Scaling of the critical current in ITER type niobium-tin superconductors in relation to the applied field, temperature and uni-axial applied strain," IEEE Trans. Appl. Superconduct., vol. 9, no. 2, pp. 161-164, 1999.

[2] B. ten Haken, A. Godeke, and H. H. J. ten Kate, "The strain dependency of the critical properties of $\mathrm{Nb}_{3} \mathrm{Sn}$ conductors," J. Appl. Phys., vol. 85, no. 6, pp. 3247-3253, 1999.

[3] A. Godeke, H. J. G. Krooshoop, H. G. Knoopers, B. ten Haken, and H. H. J. ten Kate, "Experimental verification of the temperature and strain dependence of the critical properties in $\mathrm{Nb}_{3} \mathrm{Sn}$ wires," IEEE Trans. Appl. Superconduct., vol. 11, no. 1, pp. 1526-1529, 2001.

[4] A. Godeke, B. ten Haken, and H. J. J. ten Kate, "The deviatoric strain description of the critical properties of $\mathrm{Nb}_{3} \mathrm{Sn}$ conductors," Poster Presentation N2.2-03 at EUCAS 2001, submitted for publication.

[5] L. T. Summers, M. W. Guinan, J. R. Miller, and P. A. Hahn, "A model for the prediction of $\mathrm{Nb}_{3} \mathrm{Sn}$ critical current as a function of field, temperature, strain, and radiation damage," IEEE Trans. Magn., vol. 27-2, pp. 2041-2044, 1991.

[6] B. ten Haken, A. Godeke, and H. H. J. ten Kate, "The strain dependency of ITER related $\mathrm{Nb}_{3} \mathrm{Sn}$ superconductors in the temperature range from 4 to 10 Kelvin," in Proc. MT-15 Conf., L. Liangzhen, S. Guoliao, and Y. Luguang, Eds., Beijing, China, 1998, ISBN 7-03-006 720-7/TN.204, pp. 985-988.

[7] E. J. Kramer, "Scaling laws for flux pinning in hard superconductors," J. Appl. Phys., vol. 44, pp. 1360-1370, 1973.

[8] D. Dew-Hughes, "Flux pinning mechanisms in type II superconductors," Phil. Mag., vol. 30, pp. 293-305, 1974.

[9] J. L. H. Lindenhovius, E. M. Hornsveld, A. den ouden, W. A. J. Wessel, and H. H. J. ten Kate, "Powder-in-Tube (PIT) $\mathrm{Nb}_{3} \mathrm{Sn}$ conductors for high-field magnets," IEEE Trans. Appl. Superconduct., vol. 10, no. 1, pp. 975-978, 2000.

[10] J. McDonald and E. Barzi, "A model for $J_{c}$ in granular A-15 superconductors," IEEE Trans. Appl. Superconduct., vol. 11, no. 1, pp. 3884-3887, 2001.

[11] D. O. Welch, "Alteration of the superconducting properties of A15 compounds and elementary composite superconductors by nonhydrostatic elastic strain," Adv. in Cryo. Eng., vol. 26, pp. 48-65, 1980.

[12] J. W. Ekin, "Strain scaling law for flux pinning in practical supercondutors-Part 1: Basic relationship and application to $\mathrm{Nb}_{3} \mathrm{Sn}$ conductors," Cryogenics, vol. 20, pp. 611-624, 1980. 